\title{
A Risk Score to Predict the Development of Hepatic Encephalopathy in a Population-Based Cohort of Patients with Cirrhosis
}

Elliot B. Tapper MD (1,2), Neehar Parikh MD MPH (1,2), Neil Sengupta MD (3), Jessica

Mellinger MD MSc (1), David Ratz MA (4), Anna S. Lok MD (1), Grace L. Su MD $(1,2)$

1. Division of Gastroenterology and Hepatology, University of Michigan

2. Gastroenterology Section, VA Ann Arbor Healthcare System, Ann Arbor

3. Section of Gastroenterology, University of Chicago, Chicago

4. VA Center for Clinical Management Research (CCMR), Ann Arbor

Keywords: MELD score, Portal Hypertension, Falls, Motor vehicle accidents

Emails: Parikh (ndparikh@med.umich.edu), Sengupta (nsengupta@medicine.bsd.uchicago.edu), Mellinger(jmelling@med.umich.edu), Su (gsu@umich.edu), Ratz (ratzd@med.umich.edu), Lok (aslok@umich.edu)

Corresponding author:

Elliot B. Tapper, MD

3912 Taubman, SPC 5362

1500 E Medical Center Dr

Ann Arbor, MI 48109

$\mathrm{T}:(734) 647-9252$

F: (734) 936-7392

e: etapper@umich.edu

Disclosure:

1. Elliot Tapper is the guarantor of this article

2. Roles

a. Concept: Tapper

b. Analysis: Tapper, Ratz, Su, Lok, Parikh, Sengupta Mellinger

c. Data acquisition: Tapper, Ratz, Su

d. Writing: Tapper

e. Critical revision: Lok, Su, Mellinger, Parikh, Ratz, Sengupta

3. Conflicts of interest: No authors report any conflicts of interest pertinent to this work

4. Funding: None.

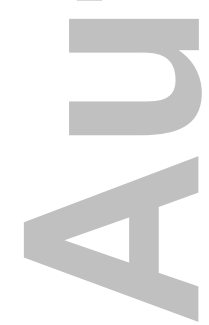

This is the author manuscript accepted for publication and has undergone full peer review but has not been through the copyediting, typesetting, pagination and proofreading process, which may lead to differences between this version and the Version of record. Please cite this article as doi:10.1002/ hep.29628. 


\begin{abstract}
Over $40 \%$ of patients with cirrhosis will develop hepatic encephalopathy (HE). HE is associated with decreased survival, falls, motor vehicle accidents, and frequent hospitalization.

Accordingly, we aimed to develop a tool to risk-stratify patients for HE development. We

studied a population-based cohort of all patients with cirrhosis without baseline $\operatorname{HE}(\mathrm{N}=1,979)$

from the Veterans Administration from Michigan, Indiana, and Ohio (1/1/2005-12/31/10) using demographic, clinical, laboratory, and pharmacy data. The primary outcome was the development of HE. Risk-scores were constructed with both baseline and longitudinal data (annually updated parameters) and validated using bootstrapping. The cohort had mean age of $58.0 \pm 8.3$ years, $36 \%$ had hepatitis C, $17 \%$ had ascites. Opiates, benzodiazepines, statins, and nonselective beta-blockers were taken at baseline by $24 \%, 13 \%, 17 \%$, and $12 \%$. Overall, 863(43.7\%) developed HE within 5 years. In multivariable models, risk factors (HR, 95\%CI) for HE included higher bilirubin (1.07, 1.05-1.09) and nonselective beta-blocker use $(1.34,1.09$ $1.64)$, while higher albumin $(0.54,0.48-0.59)$ and statin use $(0.80,0.65-0.98)$ were protective. Other clinical factors, including opiate and benzodiazepine use were not predictive. The AUROC for $\mathrm{HE}$ using the 4 significant variables in baseline and longitudinal models were $0.68(0.66$ $0.70)$ and 0.73 (0.71-0.75), respectively. Model effects were validated and converted into a risk score. A score $\leq 0$ in our longitudinal model assigns a $6 \% 1$-year probability of HE while a score $>20$ assigns a $38 \%$ 1-year risk.
\end{abstract}

Conclusion: Patients with cirrhosis can be stratified by a simple risk-score for HE that accounts for changing clinical data. Our data also highlight a role for statins in reducing cirrhosis complications including HE.

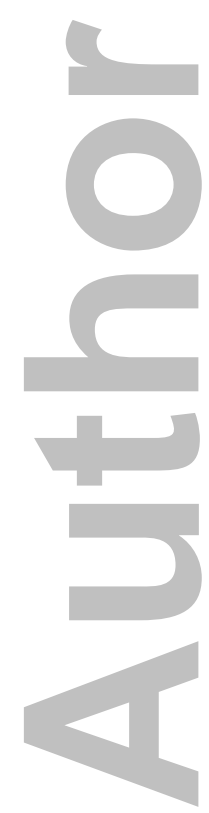


Visual Abstract (for social media)
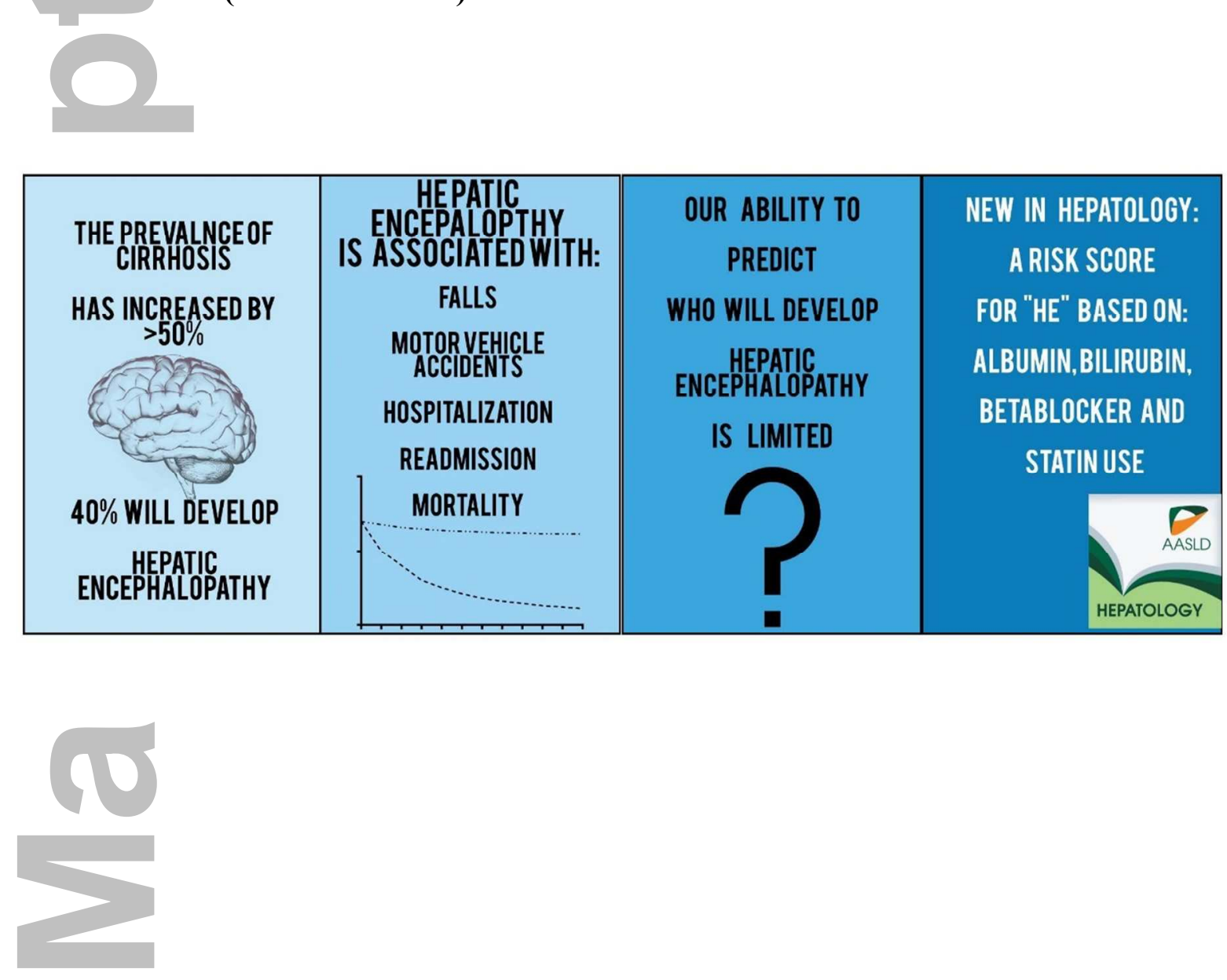

Hepatology

This article is protected by copyright. All rights reserved. 


\section{Introduction}

Cirrhosis is the final common pathway for most chronic liver diseases.(1) The majority of patients with compensated cirrhosis in the United States (US) live more than a decade after diagnosis.(2) A diagnosis of cirrhosis should prompt changes in management that include intensified treatment of the underlying disease, lifestyle changes, and counselling regarding prognosis and the risk of decompensation. Of the clinical complications of cirrhosis (variceal hemorrhage, ascites, and hepatic encephalopathy (HE)), HE is the most devastating. HE is a spectrum of reversible cognitive changes that range from mild inattention and deficits of executive function to lethargy, disorientation, and even coma. ${ }^{3-6}$ Over $40 \%$ of patients with cirrhosis will ultimately develop HE,(3) an event that is associated with decreased survival,(4) falls,(5) and motor vehicle accidents. $(6,7) \mathrm{HE}$ is also the most important factor predicting hospitalization and readmission. $(8,9)$ Following the development of HE, a patient's one-year overall mortality may rise to $>60 \%$.(10) Unfortunately, data guiding the stratification of patients with cirrhosis according to their risk of HE are limited and risk scores to predict HE development are not available. There are also no data to inform patients on how their risk changes after experiencing an improvement in liver function, for example, after cure of hepatitis $\mathrm{C}$ or alcohol abstinence. Similarly, for clinicians, there is no guidance regarding the impact on the risk of HE from common medications used in patients with cirrhosis. Herein, we analyze a large cohort of Veterans with cirrhosis followed for up to 5 years in order to determine a risk score for the development of HE and to quantify the effect of medications on the risk of HE. 


\section{Methods}

Consistent with the Food and Drug Administration's BEST (Biomarkers, EndpointS, and other Tools) terminology, we aimed to develop a risk biomarker or risk score.(11) We therefore report results of our study in accordance with the recommended framework by the Transparent reporting of a multivariable prediction model for individual prognosis or diagnosis (TRIPOD) statement (online supplement)(12) We performed a retrospective cohort study of all adult Veterans from the Veterans Affairs Integrated Service Network (VISN) 11 with cirrhosis seen in any VA facility for any outpatient or inpatient visit between 1/1/2005-12/31/2010. VISN 11 was one of the 20 integrated service networks within the VA healthcare system and provides inpatient and outpatient care for more than 685,000 Veterans within an area including Michigan, central Indiana, and northwest Ohio. In 2016, VISN 11 was absorbed into VISN 10. We used a validated definition of cirrhosis that is associated with a positive predictive value $>91 \%$ in this setting.(13) Specifically, we enrolled patients with billing codes for cirrhosis (ICD-9: 571.2, 571.5, 571.6) and an Aspartate Aminotransferase to Platelet Ratio Index (APRI) $>2.0$ or a code for one of the cirrhosis complications, including varices, ascites, or spontaneous bacterial peritonitis (456.0456.2, 572.3, 572.4, 572.8, 789.5). We excluded all patients with HE (as defined by a 572.2 code or lactulose/rifaximin prescriptions) at the time of enrollment. We included the use of lactulose or rifaximin to increase sensitivity for HE because while the 572.2 code has excellent positive predictive value $(91.5 \%-94.3 \%)$ for HE, its negative predictive value is low $(36.1 \%) .(14,15)$ Further, we limited our dataset to patients with at least 90 days of clinical follow-up. Overall, 2,747 patients had a cirrhosis diagnosis, 2,170 of whom had either a complication or an APRI > 2.0. After excluding 191 patients with an HE diagnosis at baseline, our final cohort included 
1,979 patients. The institutional review board of the Ann Arbor Veterans Administration approved the study prior to data collection.

\section{Outcome and predictors}

Our primary outcome was the development of HE. Patients were censored at the time of death, liver transplant, or loss-to-follow-up at time of the last clinical observation (in patients who did not die). Five-year survival was determined based on the VA's Beneficiary Identification Records Locator Subsystem death file. For each patient, deaths were recorded from the beginning of the study through 5 years from their index enrollment date.

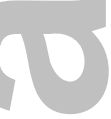

Baseline predictors of HE were defined as follows: Demographic predictors were age at diagnosis, sex, race/ethnicity, and urban vs. rural residence(16) determined based on VA Planning Systems Support Group geocoding (19). Clinical predictors included the etiology of liver disease (viral hepatitis, alcoholic, or other cirrhosis), alcohol intake at baseline (based on the AUDIT-C)(17), comorbidities (based on the Elixhauser index),(18) as well as cirrhotic complications, each defined by their corresponding ICD-9 code or procedure code (in the case of paracentesis).(Table 1) Laboratory predictors included standard parameters (albumin, total bilirubin, international normalized ratio (INR), creatinine, sodium, platelet count) as well as transformed variables such as the Model for Endstage Liver Disease (MELD).(19) Pharmacological predictors included any filled prescription ( $\geq 30$ day supply) for several classes of medication. The medication classes were selected on the basis of their association with cirrhotic conditions (diuretics/ascites, beta-blockade/varices), reported negative or positive 
associations with cirrhotic complications (statins,(20) metformin,(21) and proton pump inhibitors(22)), and psychoactive medications that may have increased risk of adverse events in patients with cirrhosis (opiates, benzodiazepines, antidepressants, and antipsychotics).(5) Medications were searched using generic and brand names, and both short and long acting formulations.(Supplemental Table 1) For the purpose of longitudinal modeling, we included updated laboratory data and interval filled prescriptions of each medication class. Statistical analyses were performed using STATA version 14 (College Station, TX) and R version v.vv (CRAN).

Analysis: Baseline data

Pearson's $\chi 2$-tests and Student's t-tests were used as appropriate for bivariate analyses of categorical and continuous predictors of HE, respectively. Multivariable Cox proportional hazard analysis was performed to determine independent predictors with candidate variables identified from univariate analyses with significant associations $(\mathrm{p}<0.1)$. Variables with missing data were not entered into final models. Age, sex and comorbidity were included in a preliminary model given biological plausibility despite a lack of statistical association. However, because these factors did not alter model performance of selected factors, they were excluded for parsimony. Based on the regression coefficients of significantly associated variables in the Cox model, a risk score was constructed using the simplest model. First, prediction accuracy was estimated using the area under the receiver operating characteristics curve (AUROC). We performed validity assessment of the model using internal validation using bootstrap.(23) Bootstrap samples are random samples drawn with replacement from the original sample. We repeatedly fitted the 
model in 10,000 bootstrap samples and evaluated its performance on the original sample in order to obtain a measure of model optimism and bias. Higher measures of optimism would suggest a risk for poorer performance in external datasets. Second, the regression coefficients of the multiple logistic regression model were used to derive a corresponding integer scoring system. $(24,25)$ Clinical variables in the final multivariable model were organized into clinically meaningful categories, each with a specific reference value. We then assigned a referent risk for each factor with the base risk assigned 0 points in the scoring system, with higher points corresponding to greater risk. Next, we calculated the difference in regression units between each category and the base category, and set the constant, B, as the number of regression units corresponding to 1 point. The points for each risk factor were calculated as the difference in regression units between each category and its base category divided by the constant. We then used the point system to divide the cohort into tertiles of risk to describe the corresponding risk of HE over a narrow range of scores.

\section{Analysis: Longitudinal data}

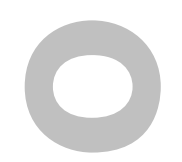

The analytic procedures for the 5-year risk of HE using baseline data described above were repeated to construct risk models using longitudinal data. In this case, clinical variables and medication utilization were updated to reflect the patient's status each year using the values (including medication fills) obtained closest to years 1, 2, 3, 4 and 5. Each year the clinical variable would be updated to reflect any changes that occurred during the prior year to predict outcomes in the following year (until an outcome occurs or the patient is censored). Accordingly, 
the longitudinal model provides a 1-year risk of HE for any given patient based on their most recent clinical parameters.

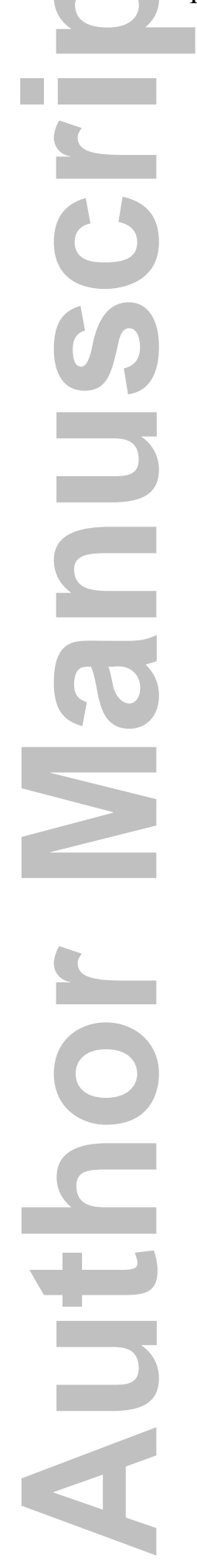


Results

Of the 1,979 patients included, the cohort had mean age of $58.0 \pm 8.3$ years, and was predominantly male (98\%) and white (74\%). Overall, 36\% had hepatitis C and 13\% were coded as having alcoholic cirrhosis. Of the 371 patients with AUDIT-C scores, 150 (40\%, all with alcoholic liver disease) scored $\geq 4$, consistent with alcohol abuse. At baseline, very few patients had received a paracentesis $(79,4 \%)$, however $350(17 \%)$ had the ascites diagnosis code; 69 $(3.5 \%)$ patients had experienced variceal hemorrhage while 228 (11.5\%) were taking nonselective beta-blockers at enrollment. Opiates, benzodiazepines, proton pump inhibitors (PPI), and statins were taken at baseline by $24 \%, 13 \%, 35 \%$, and $17 \%$. The average albumin, bilirubin, and INR were $3.40 \mathrm{~g} / \mathrm{dL}, 1.97 \mathrm{mg} / \mathrm{L}$, and 1.31, respectively. One hundred and sixty-one (8\%) had platelet counts less than $100 * 10^{9} /$ L. During follow up, $863(43.6 \%)$ patients developed overt HE. The cumulative probabilities of overt $\mathrm{HE}$ at 1,3 , and 5 years was $22.6 \%, 36.9 \%$, and $43.6 \%$, respectively. The median time to the development of HE from study enrollment was 340 days (IQR 71-842). Median survival time was 747 days for those who developed HE and 1490 days (IQR 448-1812) for those who did not develop HE. Only $16(0.8 \%)$ underwent liver transplantation during the study period.

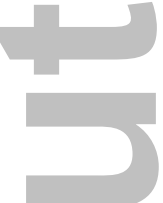

Baseline characteristics of the patients who did and those who did not develop overt HE are delineated in Table 1. Demographics, etiology of liver disease, and presence of cirrhosis complications were comparable in the two groups. Patients who developed HE had lab values 
suggesting more advanced liver disease: higher bilirubin and lower albumin values but creatinine values were lower. Baseline use of sedating, pain and anti-psychotic medications was similar, except for opiate use which was paradoxically more common in patients who did not develop HE (26.3\% vs $21.2 \%, \mathrm{P}<0.009)$. Of the medications examined, the biggest difference between the two groups was a significantly lower use of statins in patients who developed HE (13.3\% vs $19.2 \%, p=0.0005)$. Non-selective beta-blocker use was associated with increased risk of HE, ( $13 \%$ of those who developed HE versus $10.4 \%$ of those who did not develop HE; HR 1.27, 95\% CI 1.04-1.55). Of the 230 patients taking beta-blockers, the vast majority (79.6\%) were receiving propranolol.

Adjusted Associations with HE Risk Over Time

Table 2 details the associations of baseline variables with the development of HE over time in Cox proportional hazards models. Variables with significant associations on univariate analysis included the presence of ascites (by ICD code), receipt of paracentesis, total bilirubin, INR, albumin, serum sodium, and use of opiates, non-selective beta-blockers and statins. In multivariable adjustments, only baseline bilirubin (hazard ratio (HR) 1.066), albumin (HR 0.532), statin use (HR 0.795) and non-selective beta-blocker use (HR 1.338) were associated with the development of HE.

Predicting Short- and Long-Term Risk of HE 
Baseline models provide estimates of 5-year risk of HE, while longitudinal models provide an annual estimate of risk using updated parameters. The area under the receiver operating characteristic curves (AUROC) with 95\% confidence intervals (95\%CI) for the development of HE for predictive models using baseline values of the four predictors: total bilirubin, albumin, statin use, and non-selective beta-blocker use is $0.68(0.66-0.70)$. The bias obtained from this estimate in the internal validation procedure was 0.00045 (standard error 0.0095), suggesting limited optimism (i.e. after correcting the c-statistic, the result is still 0.68). When the longitudinal model for annual risk of HE was executed with the same variables, the resulting AUROC was $0.73(0.71-0.75)$. The performance of neither baseline nor longitudinal models improved when bilirubin was replaced by the MELD score (c-statistic 0.68 for both MELD and MELD-Na) or when use of other medications: proton pump inhibitors, opiates, benzodiazepines, anti-depressants, gabanergic medications singly or in combination was added to the model. (Supplementary Table 2)

Constructing a Risk Score for Short- and Long-Term Risk of HE

Risk scores using both baseline and longitudinal data are presented in Table 3 and the frequency distribution of the scores is delineated in Supplementary Figure 1. The median (range) risk scores in baseline and longitudinal models were, respectively, 8 (-23 to 62) and 9 (17 to 59 ). The risk strata for the baseline and longitudinal models were $<-10,-9$ to 20 , and $\geq 21$; and $<0,1-20$, and $\geq 21$, respectively. The proportion of patients with low, intermediate, and high baseline risk score who had developed HE is depicted in Figure 1. A Kaplan-Meier curve is presented in Figure 2 to illustrate the clear separation of risk curves between the 3 categories of risk score selected in Figure 1. In the baseline model, a score of $\leq-10$ was associated with a 5- 
year risk of $\mathrm{HE}$ of $27 \%$ while a score $>-10$ was associated with a 5 -year risk of $\mathrm{HE}>49 \%$. In the longitudinal model, which factored in changes of labs and medications, the break point for increased risk was a score of $\geq 1$. A score of $\leq 0$ was associated with a $6 \%$ risk of $\mathrm{HE}$ in the following year, while a score of $\geq 1$ was associated with a $25 \%$ risk of HE over the following year. A score $\leq 0$ in the longitudinal model carried an $89 \%(95 \%$ CI, $88-90 \%)$ negative predictive value for the development of overt HE. In the baseline model, a cutoff of $\geq-11$ provides $90.7 \%$ sensitivity and a cutoff of $\geq 27$ provides $91.2 \%$ specificity. In the longitudinal model, a cutoff of $\geq-3$ provides $90.3 \%$ sensitivity while a cutoff of $\geq 19$ provides $90.6 \%$ specificity.

Hepatology

This article is protected by copyright. All rights reserved. 


\section{Discussion}

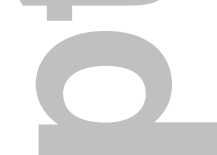

Hepatic encephalopathy (HE) is a devastating complication of cirrhosis. Accurate prognostics and preventative measures are lacking. To bridge this gap, we developed a simple, 4component risk score for HE that can be used during the routine evaluation of outpatients with cirrhosis.

\section{Why the score works}

First, two components of the risk score were low albumin and high bilirubin levels suggesting that the primary driver of HE risk is the severity of liver dysfunction. Albumin and bilirubin comprise 2 of 3 objective components of the Child-Turcotte-Pugh (CTP) score, an established measure of severity of liver disease. We did not analyze CTP score as ascertaining the severity of ascites and HE from administrative data is challenging. Furthermore, our outcome of interest is HE which is included in the CTP score. Second, we found that non-selective betablocker use was associated with increased risk of HE. Given that nonselective beta-blockade is used for primary and secondary prophylaxis of variceal hemorrhage, this finding is likely a proxy for high-risk varices or severe portal hypertension. Third, emerging data support a beneficial role of statin in cirrhosis and our findings are confirmatory. The mechanism of statin's benefit may relate to a salutary effect on portal pressures (by modulating intrahepatic endothelial dysfunction), as established in controlled studies(26). These findings have been extrapolated to explain the beneficial effects of statins on mortality and decompensation.(27, 28).(29) A metaanalysis of four observational studies examining the effects of statins on hepatic decompensation 
found a robust effect (relative risk $0.54,95 \%$ CI $0.46-0.62 ; \mathrm{I}^{2}=0 \%$ ) but no specific data on $\mathrm{HE}$ were provided.(20) Beyond portal pressure changes, statins have well known anti-inflammatory and immunomodulatory properties.(30) Since portal hypertension leads to shunting of ammonia and inflammation enhances ammonia-induced neurotoxicity,(31) the effects of statins in preventing HE is plausible.

\section{How to use the risk score}

Given the harms associated with HE, it is currently recommended that patients with cirrhosis undergo screening. Specifically, the American Association for the Study of Liver Diseases recommends patients with cirrhosis be assessed for covert HE, a precursor of overt HE characterized by executive function deficits and decreased reaction speeds.(32) The goal of this recommendation is to identify at-risk patients and provide counselling (particularly regarding driving and nutrition) and even consider pharmacotherapy (i.e. lactulose or rifaximin). Unfortunately, in order to diagnose covert HE, one must consult a neuropsychologist for batteries of psychological tests that are administered and scored against local reference data.(31) Given the complexity and cost of its assessment, most patients are not screened.(33) Though there are promising alternative methods (e.g. EncephalApp), none have been validated in clinical practice to predict clinical outcomes. In order to increase the screening of cirrhosis patients at risk for $\mathrm{HE}$, the tests used must be simple, low cost, and can be applied during routine clinical follow up. Our 4-component risk score meets these criteria. To enhance clinical utility, we provided cutoff values to maximize sensitivity or specificity according to clinical settings. In general, screening tests aim to maximize sensitivity for at-risk patients; however, there may be circumstances when we may prefer to maximize specificity to avoid mislabeling patients as 
having high risk for HE (e.g. compensated patients who report high quality of life(34)). As with the standard tests for covert HE, our score's cutoffs also create patients with borderline or indeterminate results which can be difficult to interpret clinically. These results must be evaluated in the patient's clinical context, prompting intermediate interventions (counselling and enhanced nutritional support) or viewed as a call for closer observation for decompensated patient. We provide estimates of 1-year risk in the longitudinal model for this reason. Given that neuropsychological testing is not widely available, re-defining the pre-morbid state for overt HE from covert HE to this risk score would substantially expand the tested population. Although the treatments, including improved nutrition, lactulose and rifaximin, are safe and well-tolerated, this strategy may lead to overtreatment. Additionally, future study is needed to confirm treatment response for patients with high risk scores, treating patients with covert HE often forestall the development of overt $\mathrm{HE}$ and improve quality of life.

\section{What this study adds to prior studies}

Predictors of HE have been poorly characterized and prior unadjusted estimates of HE risk offer little guidance. In a cohort of 293 patients, Gines et al found that the unadjusted risk of $\mathrm{HE}$ in an observational cohort of patients with cirrhosis at 3 years was roughly $20 \%$.(2) Later, Jepsen using a population-based cohort of Danish patients,(10) and Sangiovanni with a 17-year cohort from Milan, both showed that HE developed slowly in cirrhosis patients without prior portal hypertensive complications. $(10,35)$ Both studies neither included laboratory data in their risk estimates nor provided a risk assessment tool. In contrast, our study provides a tool to distinguish low from high risk patients using widely available markers. Dienstag and Konerman, both analyzing the HALT-C cohort (a clinical trial of patients receiving long-term interferon for 
advanced hepatitis C), and Gomez et al, analyzing an observational cohort from Cuba, both classified risk of decompensation as a composite outcome (including HE) with models that included CTP score and platelet count.(36-38) Unfortunately, the number of HE events in both studies (a combined 80 events out of 1400 patients),(36, 37) limited the ability to provide accurate prediction of HE. Moreover, prior studies did not provide tools to assess changing risk during follow-up. Patients at high risk for HE should be offered a suite of clinical and lifestyle changes to promote improved liver function. At a minimum, this includes the eradication of hepatitis $\mathrm{C}$ if present,(39) intensified treatment for alcohol abuse,(40) and specific guidance on nutritional intake (i.e. $\geq 1.25 \mathrm{~g} / \mathrm{kg}$ protein daily),(32) each of which is associated with improved liver function (i.e. albumin and/or bilirubin). Additionally, there may be a chemopreventive role for statins. The principal advance of our simple, HE-specific risk score is that it can provide revised estimates as the patient's liver function and other parameters such as use of statin changes.

Interpreting Results in the Context of the Study Design

The strengths of our study include a large cohort of patients with data on lab values and medications. We used a previously validated algorithm for identifying patients with cirrhosis within the VA system.(13) We analyzed not only baseline data but also longitudinal data simulating the effects of incident drug prescriptions and changes in lab values in prospective studies. However, there are several limitations that are inherent in retrospective studies. First, our requirement for an APRI $>2.0$ or a cirrhotic complication, in addition to cirrhosis diagnosis codes was aimed to ensure specificity of the diagnosis of cirrhosis but it may have enriched the cohort with more advanced cirrhosis and higher risk of HE. compared to other studies of 
compensated patients. $(36,37)$ Second, the VA patient population is predominantly male. Third, any retrospective data is subject to the risk of unmeasured confounders. Some factors that have been suggested to play a role in the development of HE such as baseline educational attainment (reflecting cognitive reserve)(34) and sarcopenia (muscle actively metabolizes ammonia),(31) could not be examined in this study. Fourth, while high bilirubin and low albumin reflecting severity of liver disease are likely causally related to development of HE, other predictors may not be causally related. Fifth, some of our data on the effects of specific drug classes conflict with prior reports. Our data from outpatients with cirrhosis suggests that after adjusting for disease severity, psychoactive medications, metformin, and PPI are not associated with the development of HE. $(5,21,22)$ These differences can be reconciled. Many medications possess a narrow therapeutic index in cirrhosis. Psychoactive drugs and PPI may have incremental toxicity for acutely ill hospitalized patients but limited risk-adjusted adverse effects in relatively stable outpatients. Similarly, though not tested here, we suspect that our finding of the salutary effect of statins on HE risk is less likely to be observed in a cohort of infected, acutely decompensated hospitalized patients. The beneficial effect of metformin, a known modifier of glutaminase activity in vitro,(21) was established in a cohort of 80 patients with diabetes. Our sample size was larger, including roughly 2.5 times the number of metformin users, and by including nondiabetic patients, our cohort is fundamentally different. Finally, propranolol made up the majority of betablocers used. While these were likely proxies for clinically significant portal hypertension, we cannot be sure these data generalize to, say, carvedilol.

Conclusion 
In summary, we found that the risk of HE in patients with cirrhosis can be stratified by two readily available lab tests and a brief inventory of the medication list. Our risk score needs to be validated prospectively in external cohorts. Finally, the potential benefits of statins in preventing HE need to be studied in rigorously designed randomized controlled trials. This is particularly important for patients with cirrhosis for whom there is no effective treatment to eliminate or control the underlying cause.
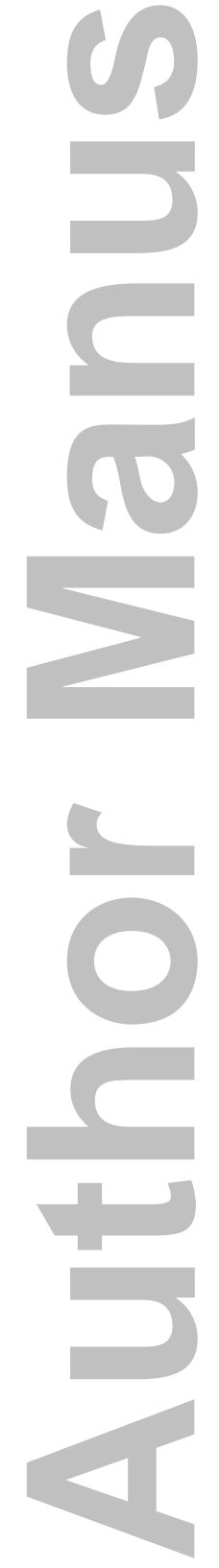
Table 1: Cohort Characteristics

\begin{tabular}{|c|c|c|c|}
\hline & No HE $(n=1116)$ & HE $(n=863)$ & P Value \\
\hline Age & $58.6(9.13)$ & $57.3(7.83)$ & .001 \\
\hline Male, n (\%) & $1096(98.2)$ & $852(98.7)$ & 0.36 \\
\hline \multicolumn{4}{|l|}{ Racelethnicity, n (\%) } \\
\hline White, not Hispanic & $814(72.9)$ & $652(75.6)$ & \multirow{4}{*}{0.12} \\
\hline White, Hispanic ethnicity & $12(1.1)$ & $16(1.9)$ & \\
\hline Black & $217(19.3)$ & $154(17.8)$ & \\
\hline Other & $73(6.5)$ & $41(4.8)$ & \\
\hline Urban/rural status: Urban, $n(\%)$ & $741(66.4)$ & $586(67.9)$ & 0.48 \\
\hline Elixhauser comorbidity, mean (s.d.) & $3.62(2.47)$ & $3.10(2.30)$ & 0.24 \\
\hline Hepatitis C, $n(\%)$ & $395(35.4)$ & $312(36.2)$ & 0.73 \\
\hline Alcoholic Cirrhosis, $n(\%)$ & $157(14.1)$ & $103(11.9)$ & 0.16 \\
\hline Variceal bleed, $n(\%)$ & $33(3.0)$ & $36(4.2)$ & 0.14 \\
\hline Hepatocellular carcinoma, $n(\%)$ & $33(3.0)$ & $17(2.0)$ & 0.17 \\
\hline Ascites, $n(\%)$ & $194(17.4)$ & $146(16.9)$ & 0.79 \\
\hline Paracentesis, $n(\%)$ & 44 (3.9) & $35(4.1)$ & 0.90 \\
\hline \multicolumn{4}{|l|}{ Labs } \\
\hline INR mean (s.d.) & $1.29(0.70)$ & $1.34(0.59)$ & 0.22 \\
\hline Thrombocytopenia, $n(\%)$ & $90(8.1)$ & $71(8.2)$ & 0.90 \\
\hline Creatinine, $\mathrm{mg} / \mathrm{dL}$ mean (s.d.) & $1.13(0.83)$ & $1.02(0.68)$ & 0.002 \\
\hline Bilirubin (mg/dl), mean (s.d.) & $1.66(2.42)$ & $2.37(3.23)$ & $<0.0001$ \\
\hline Albumin, g/dL mean (s.d.) & $3.49(0.73)$ & $3.27(0.70)$ & $<.0001$ \\
\hline Sodium, meq/L mean (s.d.) & $137.8(4.38)$ & $137.3(8.28)$ & 0.09 \\
\hline \multicolumn{4}{|l|}{ Medication use } \\
\hline Benzodiazepine use, $n(\%)$ & $145(13.0)$ & $107(12.4)$ & 0.69 \\
\hline Gabanergic use, $n(\%)$ & $88(7.9)$ & $61(7.1)$ & 0.49 \\
\hline Opiate use, $n(\%)$ & $293(26.3)$ & $183(21.2)$ & 0.009 \\
\hline Antipsychotic use, $n(\%)$ & $83(7.4)$ & $64(7.4)$ & 0.99 \\
\hline Proton Pump Inhibitor use, $n(\%)$ & $404(36.2)$ & $297(34.4)$ & 0.41 \\
\hline Anti-depressant use, $n(\%)$ & $279(25.0)$ & $218(25.3)$ & 0.89 \\
\hline Tricyclic Anti-depressant use, $n(\%)$ & $140(12.5)$ & $116(13.4)$ & 0.56 \\
\hline Diuretic use, $n(\%)$ & $463(41.5)$ & $324(37.5)$ & 0.08 \\
\hline Metformin use, n \%) & $95(11.0)$ & $129(11.6)$ & 0.29 \\
\hline Nonselective Betablocker use, $n(\%)$ & $116(10.4)$ & $112(13.0)$ & 0.07 \\
\hline Statin use, $n(\%)$ & $214(19.2)$ & $115(13.3)$ & 0.0005 \\
\hline
\end{tabular}


Table 2: Univariate and Multivariate Associations with Hepatic Encephalopathy

\begin{tabular}{|c|c|c|c|c|}
\hline & \multicolumn{2}{|r|}{ Unadjusted } & \multicolumn{2}{|r|}{ Adjusted } \\
\hline & $\begin{array}{c}\text { Hazard } \\
\text { ratio }\end{array}$ & 95\% CI (P value) & $\begin{array}{c}\text { Hazard } \\
\text { ratio }\end{array}$ & 95\% CI P value \\
\hline Age & 1.005 & $0.996-1.013(0.27)$ & & \\
\hline Male gender & 1.486 & $0.820-2.692(0.19)$ & & \\
\hline Urban location & 1.063 & $0.921-1.226(0.40)$ & & \\
\hline Hepatitis C & 0.894 & $0.778-1.027(0.11)$ & & \\
\hline Alcoholic Cirrhosis & 1.087 & $0.885-1.336(0.43)$ & & \\
\hline Thrombocytopenia & 1.084 & $0.850-1.382(0.51)$ & & \\
\hline Hepatitis B & 0.977 & $0.596-1.602(0.93)$ & & \\
\hline Variceal bleed & 1.346 & $0.964-1.879(0.08)$ & & \\
\hline Hepatorenal syndrome & 0.332 & $0.047-2.353(0.27)$ & & \\
\hline $\begin{array}{l}\text { Hepatocellular } \\
\text { carcinoma }\end{array}$ & 1.069 & $0.661-1.728(0.79)$ & & \\
\hline Ascites & 1.480 & $1.238-1.769(<.0001)$ & & \\
\hline Paracentesis & 1.612 & $1.149-2.262(0.006)$ & & \\
\hline Bilirubin (mg/dl) & 1.103 & $1.086-1.121(<.0001)$ & 1.068 & $1.048-1.088(<.0001)$ \\
\hline $\begin{array}{l}\text { International normalized } \\
\text { ratio }\end{array}$ & 1.124 & $1.041-1.213(0.003)$ & & \\
\hline Creatinine & 0.953 & $0.853-1.064(0.39)$ & & \\
\hline Albumin & 0.501 & $0.457-0.549(<.0001)$ & 0.543 & $0.493-0.597(<.0001)$ \\
\hline Sodium & 0.985 & $0.978-0.992(<.0001)$ & & \\
\hline Benzodiazepine & 0.877 & $0.717-1.074(0.21)$ & & \\
\hline Gabanergic & 0.907 & $0.699-1.176(0.46)$ & & \\
\hline Opiate & 0.794 & $0.674-0.934(0.006)$ & & \\
\hline Antipsychotic & 0.932 & $0.723-1.203(0.59)$ & & \\
\hline Proton Pump Inhibitor & 0.938 & $0.815-1.079(0.37)$ & & \\
\hline Anti-depressant & 0.908 & $0.778-1.058(0.22)$ & & \\
\hline $\begin{array}{l}\text { Tricyclic Anti- } \\
\text { depressant }\end{array}$ & 1.005 & $0.826-1.222(0.96)$ & & \\
\hline Diuretic & 0.943 & $0.821-1.082(0.40)$ & & \\
\hline Betablocker & 1.235 & $1.013-1.507(0.04)$ & 1.268 & $1.036-1.551(0.02)$ \\
\hline Statin & 0.742 & $0.610-0.903(0.003)$ & 0.740 & $0.608-0.901(0.003)$ \\
\hline
\end{tabular}

Variables that retained statistically significant associations in multivariable models are included in the rightmost columns and subsequently entered into the final model for risk score construction. 
Table 3: Construction of a Risk Score for Hepatic Encephalopathy

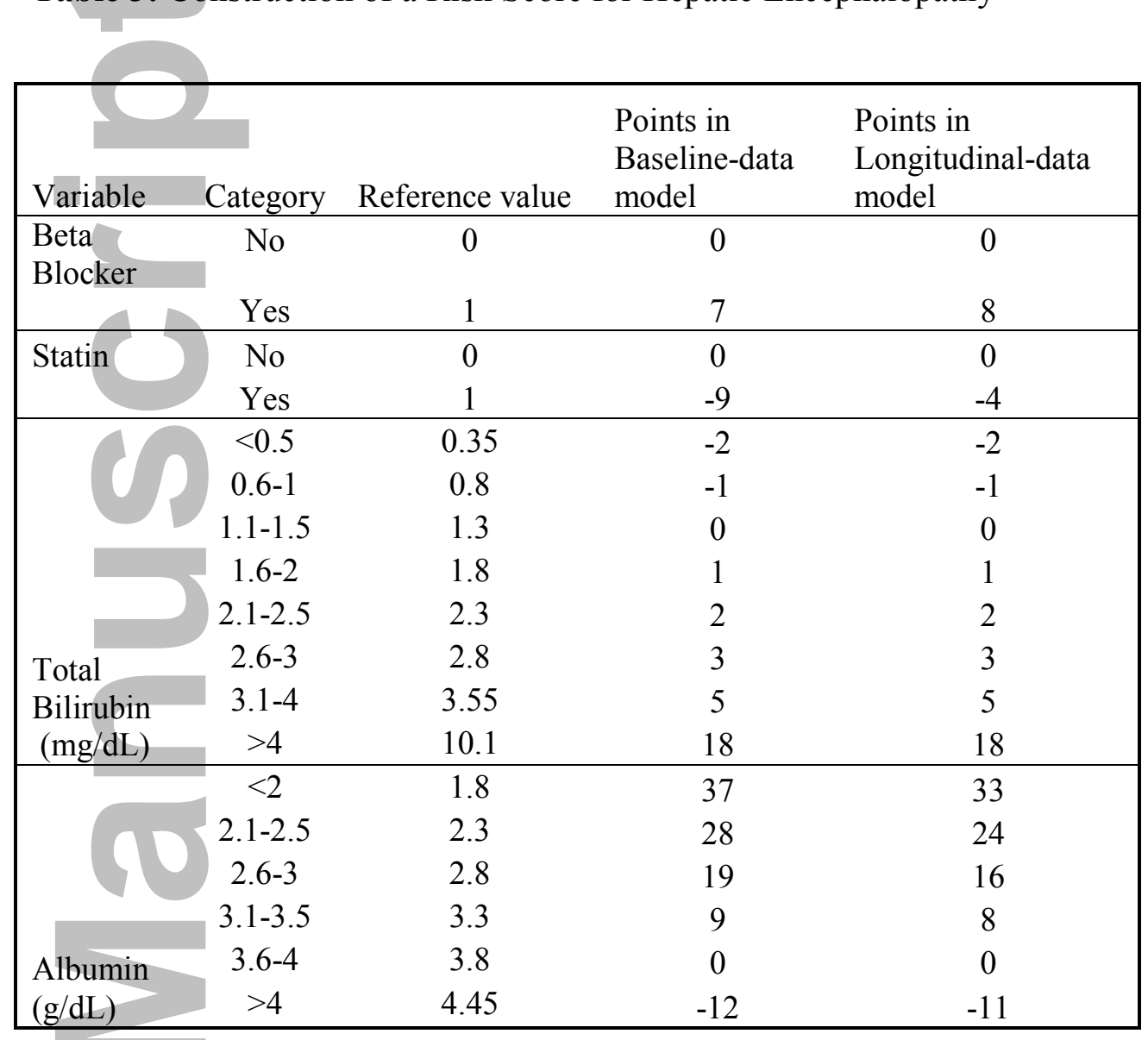




\section{FIGURES}

Figure 1: Proportion of Patients Developing HE According to their Risk Score

Left: The proportion of patients who develop HE over the course of 5 years follow-up, stratified by risk score category.

Right: The proportion of patients who develop HE over the following year in a model using longitudinal data, stratified by risk score.

\section{Figure 2: Risk of HE from Baseline Assessment by Risk Strata}

Low risk patients have baseline risk scores of $\leq-10$, Intermediate risk patients have scores between -9 and 20, and high risk patients have scores $>20$.
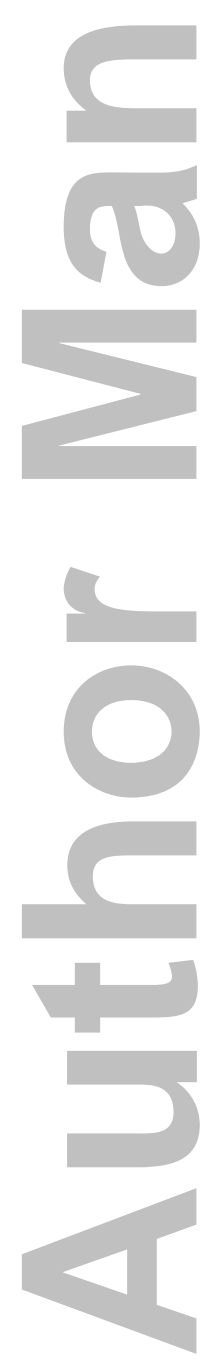


\section{References}

1. Schuppan D, Afdhal NH. Liver cirrhosis. Lancet 2008;371:838-851.

2. Gines P, Quintero E, Arroyo V, Teres J, Bruguera M, Rimola A, Caballeria J, et al. Compensated cirrhosis: natural history and prognostic factors. Hepatology 1987;7:122-128.

3. D'Amico G, Garcia-Tsao G, Pagliaro L. Natural history and prognostic indicators of survival in cirrhosis: a systematic review of 118 studies. Journal of hepatology 2006;44:217-231.

4. Wong RJ, Gish RG, Ahmed A. Hepatic encephalopathy is associated with significantly increased mortality among patients awaiting liver transplantation. Liver Transplantation 2014;20:1454-1461.

5. Tapper EB, Risech-Neyman Y, Sengupta N. Psychoactive medications increase the risk of falls and fall-related injuries in hospitalized patients with cirrhosis. Clinical Gastroenterology and Hepatology 2015;13:1670-1675.

6. Bajaj JS, Hafeezullah M, Franco J, Varma RR, Hoffmann RG, Knox JF, Hischke D, et al. Inhibitory control test for the diagnosis of minimal hepatic encephalopathy. Gastroenterology 2008;135:15911600 e1591.

7. Bajaj JS, Hafeezullah M, Hoffmann RG, Varma RR, Franco J, Binion DG, Hammeke TA, et al. Navigation skill impairment: Another dimension of the driving difficulties in minimal hepatic encephalopathy. Hepatology 2008;47:596-604.

8. Tapper EB, Finkelstein D, Mittleman MA, Piatkowski G, Chang M, Lai M. A Quality Improvement Initiative Reduces 30-Day Rate of Readmission for Patients With Cirrhosis. Clinical Gastroenterology and Hepatology 2015.

9. Tapper EB, Halbert B, Mellinger J. Rates of and Reasons for Hospital Readmissions in Patients with Cirrhosis: A Multistate Population-based Cohort Study. Clinical Gastroenterology and Hepatology 2016.

10. Jepsen P, Ott P, Andersen PK, Sørensen HT, Vilstrup H. Clinical course of alcoholic liver cirrhosis: A Danish population-based cohort study. Hepatology 2010;51:1675-1682.

11. Group F-NBW. BEST (Biomarkers, EndpointS, and other Tools) Resource. 2016.

12. Moons KG, Altman DG, Reitsma JB, loannidis JP, Macaskill P, Steyerberg EW, Vickers AJ, et al. Transparent Reporting of a multivariable prediction model for individual Prognosis or diagnosis (TRIPOD): Explanation and ElaborationThe TRIPOD Statement: explanation and elaboration. Annals of internal medicine 2015;162:W1-W73.

13. Kanwal F, Kramer JR, Buchanan P, Asch SM, Assioun Y, Bacon BR, Li J, et al. The quality of care provided to patients with cirrhosis and ascites in the Department of Veterans Affairs. Gastroenterology 2012;143:70-77.

14. Nehra MS, Ma Y, Clark C, Amarasingham R, Rockey DC, Singal AG. Use of administrative claims data for identifying patients with cirrhosis. Journal of clinical gastroenterology 2013;47:e50.

15. Goldberg D, Lewis J, Halpern S, Weiner M, Lo Re V. Validation of three coding algorithms to identify patients with end-stage liver disease in an administrative database. Pharmacoepidemiology and drug safety 2012;21:765-769.

16. West AN, Lee RE, Shambaugh-Miller MD, Bair BD, Mueller KJ, Lilly RS, Kaboli PJ, et al. Defining "rural" for veterans' health care planning. The Journal of Rural Health 2010;26:301-309.

17. Bush K, Kivlahan DR, McDonell MB, Fihn SD, Bradley KA. The AUDIT alcohol consumption questions (AUDIT-C): an effective brief screening test for problem drinking. Archives of internal medicine 1998;158:1789-1795.

18. Elixhauser A, Steiner C, Harris DR, Coffey RM. Comorbidity measures for use with administrative data. Medical care 1998;36:8-27.

19. Kamath PS, Wiesner RH, Malinchoc M, Kremers W, Therneau TM, Kosberg CL, D'Amico G, et al. A model to predict survival in patients with end-stage liver disease. Hepatology 2001;33:464-470. 
20. Kim RG, Loomba R, Prokop LJ, Singh S. Statin Use and Risk of Cirrhosis and Related Complications in Patients with Chronic Liver Diseases: a Systematic Review and Meta-analysis. Clinical Gastroenterology and Hepatology 2017.

21. Ampuero J, Ranchal I, Nunez D, del Mar Díaz-Herrero M, Maraver M, del Campo JA, Rojas A, et al. Metformin inhibits glutaminase activity and protects against hepatic encephalopathy. PLoS One 2012;7:e49279.

22. Bajaj JS, Reddy KR, Tandon P, Wong F, Kamath PS, Garcia-Tsao G, Maliakkal B, et al. The 3-month readmission rate remains unacceptably high in a large North American cohort of patients with cirrhosis. Hepatology 2016;64:200-208.

23. Steyerberg EW, Harrell FE, Borsboom GJ, Eijkemans M, Vergouwe Y, Habbema JDF. Internal validation of predictive models: efficiency of some procedures for logistic regression analysis. Journal of clinical epidemiology 2001;54:774-781.

24. Sullivan LM, Massaro JM, D'Agostino RB. Presentation of multivariate data for clinical use: The Framingham Study risk score functions. Statistics in medicine 2004;23:1631-1660.

25. Sengupta N, Tapper EB. Derivation and Internal Validation of a Clinical Prediction Tool for 30Day Mortality in Lower Gastrointestinal Bleeding. The American journal of medicine 2017;130:601. e601-601. e608.

26. Abraldes JG, Albillos A, Bañares R, Turnes J, González R, García-Pagán JC, Bosch J. Simvastatin lowers portal pressure in patients with cirrhosis and portal hypertension: a randomized controlled trial. Gastroenterology 2009;136:1651-1658.

27. Huang Y-W, Lee C-L, Yang S-S, Fu S-C, Chen Y-Y, Wang T-C, Hu J-T, et al. Statins reduce the risk of cirrhosis and its decompensation in chronic hepatitis B patients: a nationwide cohort study. The American journal of gastroenterology 2016.

28. Abraldes JG, Villanueva C, Aracil C, Turnes J, Hernandez-Guerra M, Genesca J, Rodriguez M, et al. Addition of simvastatin to standard therapy for the prevention of variceal rebleeding does not reduce rebleeding but increases survival in patients with cirrhosis. Gastroenterology 2016;150:1160-1170. e1163.

29. Mohanty A, Tate JP, Garcia-Tsao G. Statins Are Associated With a Decreased Risk of Decompensation and Death in Veterans With Hepatitis C-Related Compensated Cirrhosis. Gastroenterology 2016;150:430-440. e431.

30. Marrone G, Maeso-Díaz R, García-Cardena G, Abraldes JG, García-Pagán JC, Bosch J, GraciaSancho J. KLF2 exerts antifibrotic and vasoprotective effects in cirrhotic rat livers: behind the molecular mechanisms of statins. Gut 2015;64:1434-1443.

31. Tapper EB, Jiang ZG, Patwardhan VR. Refining the ammonia hypothesis: a physiology-driven approach to the treatment of hepatic encephalopathy. In: Mayo Clinic Proceedings; 2015: Elsevier; 2015. p. 646-658.

32. Vilstrup H, Amodio P, Bajaj J, Cordoba J, Ferenci P, Mullen KD, Weissenborn K, et al. Hepatic encephalopathy in chronic liver disease: 2014 Practice Guideline by the American Association for the Study Of Liver Diseases and the European Association for the Study of the Liver. Hepatology 2014;60:715-735.

33. Bajaj JS, Etemadian A, Hafeezullah M, Saeian K. Testing for minimal hepatic encephalopathy in the United States: an AASLD survey. Hepatology 2007;45:833-834.

34. Patel AV, Wade JB, Thacker LR, Sterling RK, Siddiqui MS, Stravitz RT, Sanyal AJ, et al. Cognitive reserve is a determinant of health-related quality of life in patients with cirrhosis, independent of covert hepatic encephalopathy and model for end-stage liver disease score. Clinical Gastroenterology and Hepatology 2015;13:987-991. 
35. Sangiovanni A, Prati GM, Fasani P, Ronchi G, Romeo R, Manini M, Del Ninno E, et al. The natural history of compensated cirrhosis due to hepatitis C virus: A 17-year cohort study of 214 patients. Hepatology 2006;43:1303-1310.

36. Gomez EV, Rodriguez YS, Bertot LC, Gonzalez AT, Perez YM, Soler EA, Garcia AY, et al. The natural history of compensated HCV-related cirrhosis: a prospective long-term study. Journal of hepatology 2013;58:434-444.

37. Dienstag JL, Ghany MG, Morgan TR, Di Bisceglie AM, Bonkovsky HL, Kim HY, Seeff LB, et al. A prospective study of the rate of progression in compensated, histologically advanced chronic hepatitis $C$. Hepatology 2011;54:396-405.

38. Konerman MA, Zhang Y, Zhu J, Higgins PD, Lok AS, Waljee AK. Improvement of predictive models of risk of disease progression in chronic hepatitis $C$ by incorporating longitudinal data. Hepatology 2015;61:1832-1841.

39. Tapper EB, Hughes MS, Buti M, Dufour J-F, Flamm S, Firdoos S, Curry MP, et al. The optimal timing of hepatitis $C$ therapy in transplant eligible patients with Child $B$ and $C$ Cirrhosis: $A$ CostEffectiveness Analysis. Transplantation 2017.

40. Lackner C, Spindelboeck W, Haybaeck J, Douschan P, Rainer F, Terracciano L, Haas J, et al. Histological parameters and alcohol abstinence determine long-term prognosis in patients with alcoholic liver disease. Journal of Hepatology 2017;66:610-618.

Hepatology

This article is protected by copyright. All rights reserved. 

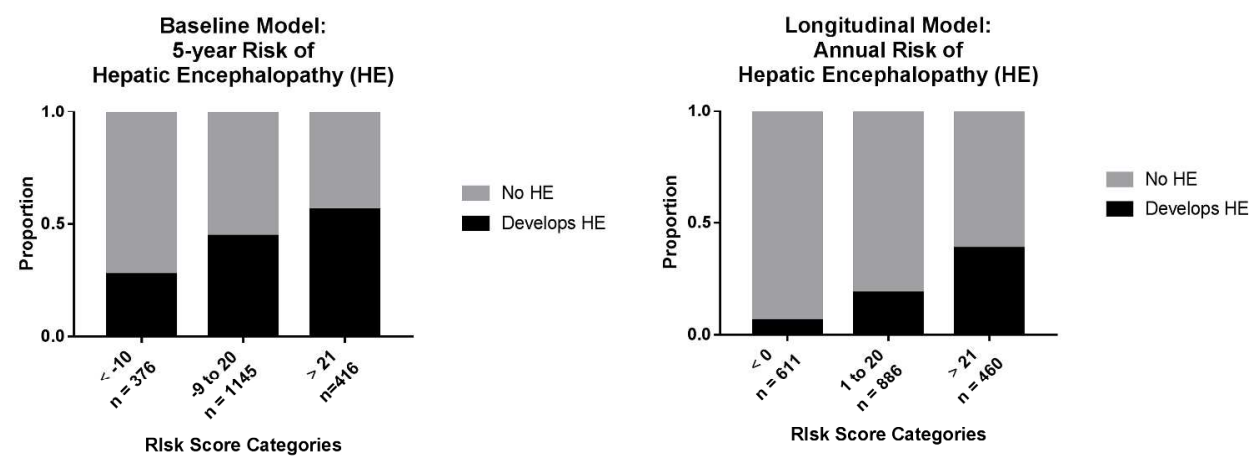

Figure 1: Proportion of Patients Developing HE According to their Risk Score

Left: The proportion of patients who develop HE over the course of 5 years follow-up, stratified by risk score category.

Right: The proportion of patients who develop HE over the following year in a model using longitudinal data, stratified by risk score.

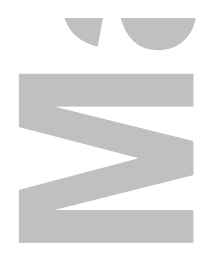

$259 \times 106 \mathrm{~mm}(300 \times 300 \mathrm{DPI})$ 


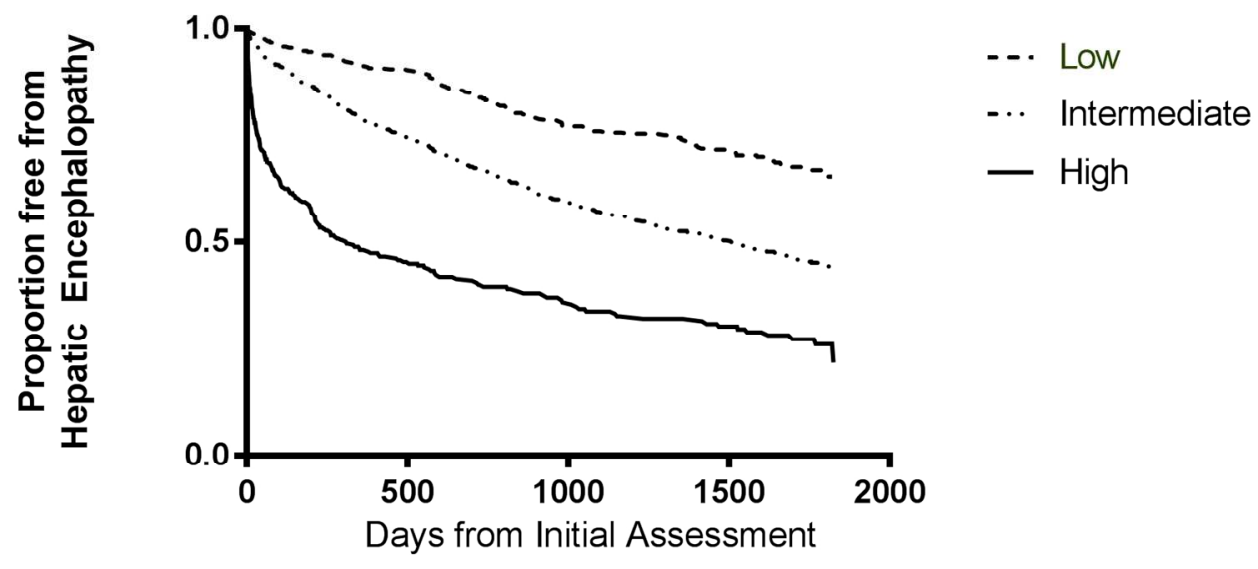

Figure 2: Risk of HE from Baseline Assessment by Risk Strata

Low risk patients have baseline risk scores of $<-10$, Intermediate risk patients have scores between -9 and 20 , and high risk patients have scores $>20$.

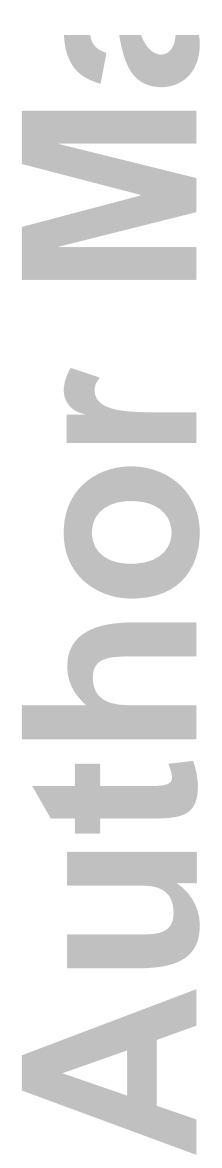

$154 \times 73 \mathrm{~mm}(300 \times 300$ DPI $)$ 
Supplementary Table 1: Medication Classes

\begin{tabular}{|c|c|c|c|c|}
\hline Benzodiazepines & Gabanergic & Antipsychotic & Opiates & Antidepressants \\
\hline Chlordiazepoxide & Gabapentin & aripiprazole & Oxycodone & citalopram \\
\hline Diazepam & Pregabalin & chlorpromazine & Morphine & bupropion \\
\hline Lorazepam & & clozapine & Hydromorphone & venlafaxine \\
\hline Oxazepam & & fluphenazine & Codeine & desvenlafaxine \\
\hline Alprazolam & & Haloperidol & Hydrocodone & escitalopram \\
\hline Clonazepam & & lurasidone & Fentanyl & fluoxetine \\
\hline Eszopiclone & & Olanzapine & Methadone & citalopram \\
\hline Zolpidem & & Quietapine & & paroxetine \\
\hline Temazepam & & Risperidal & & vilazodone \\
\hline Triazolam & & $\begin{array}{l}\text { Thioridazine } \\
\text { ziprasidone }\end{array}$ & & $\begin{array}{l}\text { sertraline } \\
\text { duloxetine }\end{array}$ \\
\hline & & & & Fluvoxamine \\
\hline & & & & Mirtazapine \\
\hline & & & & Nefazodone \\
\hline Tricyclics & Statins & Diuretic & $\begin{array}{l}\text { Proton pump } \\
\text { inhibitor }\end{array}$ & Beta blocker \\
\hline Trazodone & Atorvastatin & Furosemide & Pantoprazole & Nadolol \\
\hline Amitryptiline & Rosuvastatin & Spironolactone & Omeprazole & Propranolol \\
\hline Nortryptiline & Pravastatin & Amiloride & Rabeprazole & Carvedilol \\
\hline Desipramine & Lovastatin & Hydrochlorothiazide & Lansoprazole & \\
\hline Imipramine & Simvastatin & Epleronone & Esomeprazole & \\
\hline Doxepin & & $\begin{array}{l}\text { Chlorthalidone } \\
\text { Indapamide }\end{array}$ & Deslansoprazole & \\
\hline & & Torsemide & & \\
\hline
\end{tabular}

In supplementary table 1, the medication classes explored as exposures for HE risk are detailed by their constituent medications. Both generic and brand names were searched in the clinical data warehouse.

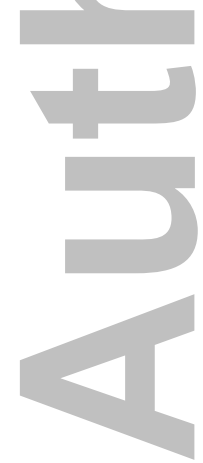


Supplementary Table 2: Incremental Changes After Including Additional Variables in Baseline and Longitudinal Risk Models

\begin{tabular}{|c|c|c|}
\hline & $\begin{array}{l}\text { Baseline only } \\
\text { AUC }(95 \% \mathrm{CI})\end{array}$ & $\begin{array}{l}\text { Longitudinal model } \\
\text { AUC (95\%CI) }\end{array}$ \\
\hline $\begin{array}{l}\text { Final model (based on Table 2) } \\
\text { (albumin, bilirubin, statin, non- } \\
\text { selective beta-blocker) }\end{array}$ & $0.68(0.66-0.70)$ & $0.73(0.71-0.75)$ \\
\hline Plus proton pump inhibitor use & $0.68(0.66-0.70)$ & $0.73(0.71-0.75)$ \\
\hline Plus benzodiazepine use & $0.68(0.66-0.70)$ & $0.73(0.71-0.75)$ \\
\hline Plus opiate use & $0.68(0.66-0.70)$ & $0.73(0.71-0.75)$ \\
\hline Plus antidepressant use & $0.68(0.66-0.70)$ & $0.73(0.71-0.75)$ \\
\hline Plus gabanergic use & $0.68(0.66-0.70)$ & $0.73(0.71-0.75)$ \\
\hline Plus all meds & $0.68(0.66-0.70)$ & $0.72(0.70-0.74)$ \\
\hline
\end{tabular}

In Supplementary Table 2, the area under the receiver operating curve (AUC) is presented for the variables selected in table 2 for both baseline and longitudinal models. We demonstrate the lack of increased risk discrimination associated with use of selected medication classes either at baseline or during follow-up (longitudinal model) 


\section{Supplementary Figure 1}
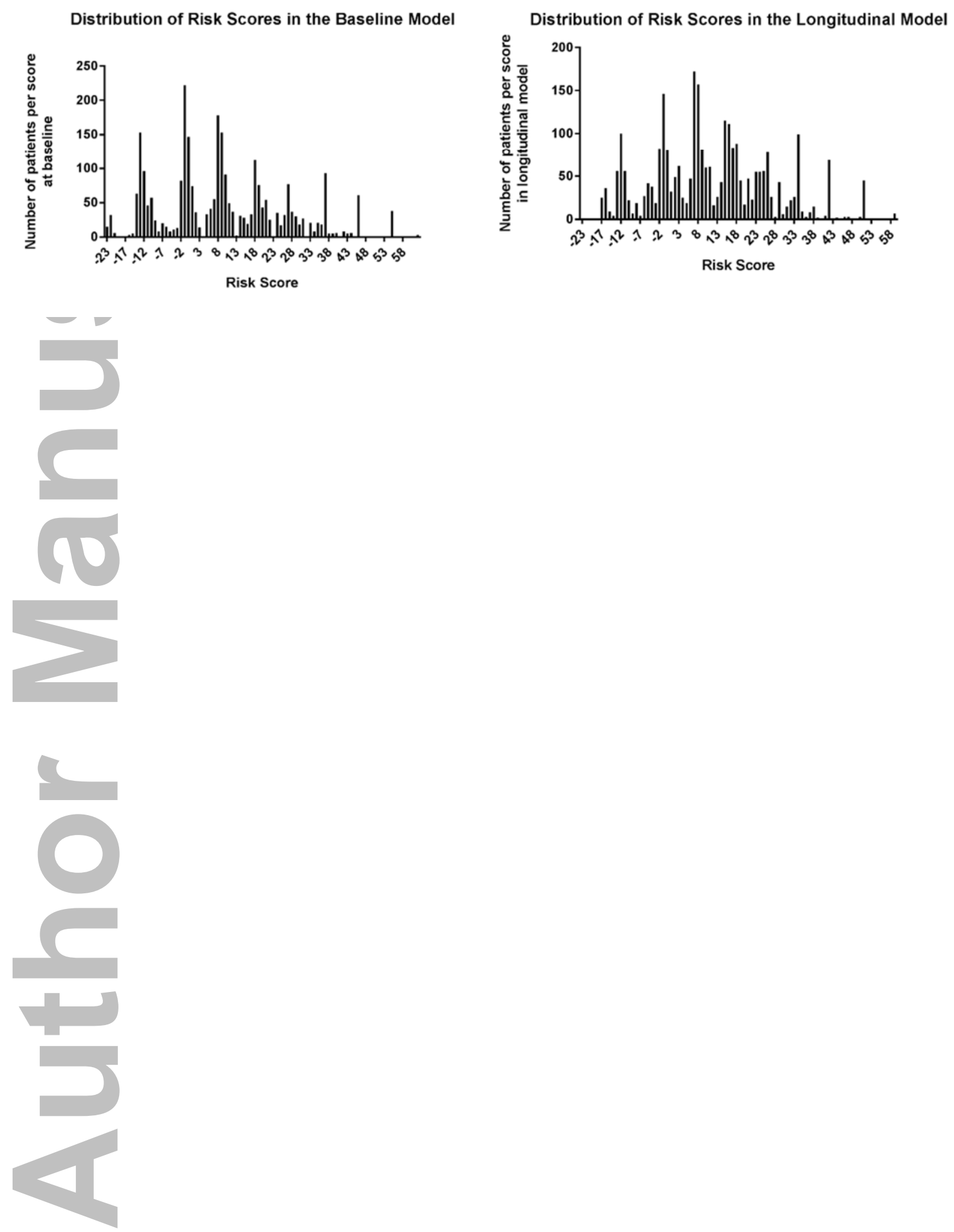